\title{
Update from RCP Quality Improvement: Improving improvement through practice, learning and research
}

The most cited article from Future Healthcare Journal is Mary Dixon Woods' paper 'Does quality improvement improve quality'. The conclusion of this article, her Harveian lecture and the recent $B M J$ paper is that it depends how it's done, and we need better research. ${ }^{2,3}$ So we need to improve how we do improvement, both now and in the future.

\section{Being rigorous but inclusive with a method}

What do we mean by quality improvement (QI)? There is no single definition. The Health Foundation states that quality improvement 'aim[s] to bring about a measurable improvement by applying specific methods within a healthcare setting ... [with] common approaches used to improve quality.' Batalden and Davidoff describe the key elements of quality improvement as understanding systems, processes, variation and context, and then developing new knowledge by testing and adapting planned changes, while measuring the effect over time. ${ }^{5}$ This needs to happen in a culture of learning as teams and organisations.

Many individuals make valiant efforts to improve quality by changing processes of care, but without applying a 'method'. If we look at the parallel with clinical practice we may change our clinical practice over time, based on context and experience but it is still grounded in core clinical methods. It is essential that individuals and teams understand and develop the skills of a basic improvement method, as they would with a clinical method. This can be understood in a classroom but is learned though application. Organisations will base their quality improvement training and practice on a core method such as the Model for improvement, Lean and Six Sigma. The main elements as described by Batalden and Davidoff should be present in whichever method is used. Most improvement practitioners have learned through practice and experience that it is necessary to add elements of other methods to their core method.

Skills to do core methods come with experience and practice, so working in teams supported by QI experts is important. Even seemingly basic methods like process mapping; plan, do, study, act; and time series measurement are not easy to do well. ${ }^{6,7}$

\section{Understanding the system}

If clinicians see a problem, we try to find a quick fix to it. Commonly, solutions are tested before the problem is understood in quality improvement projects. Spending time understanding the system in which the problem has arisen, and how it's working to produce this result is vital. This needs to explore people, behaviours, attitudes, functions, processes, variation and influencing factors. Then and only then can we build the right team to set an aim and test a hypothesis of what may result in improvement.

\section{Better measurement and evaluation}

Testing and adapting changes before we have a measurement plan in place is also a common problem. That's because measurement of how a system is working is difficult. Working more closely with measurement experts will help. The expertise of healthcare analysts are underused in healthcare improvement. Using qualitative measurement is as important as quantitative measures, yet how we capture staff or patient experience or measure culture is not well developed for quality improvement projects and programmes. We should be learning from health services research and organisational development. Evaluating the process and experience of doing quality improvement is also important so that we can adapt it and improve it in the future. This should be a feature of any publications on improvement projects or programmes as stated in SQUIRE 2.0 guidelines. ${ }^{8}$

\section{Embedded in a quality management system}

To improve and maintain quality, quality improvement approaches are not enough. These must sit as part of a quality management system that includes quality assurance/control, quality planning and quality improvement all joined together around a programme of priorities, skill development and a culture that enables and celebrates learning and leadership by all. This means it's embedded in how the organisation works and that all departments support the programmes. ${ }^{9}$

\section{Spread and adaptation rather than new approaches}

Developing new local approaches to delivering health and care is commonly the focus of improvement programmes, despite the fact that all over the country, world or even in other departments in the same organisation changes have been made that have demonstrated improvement. So, spreading and adapting good practice should perhaps be our focus, and may have more rapid impact. Our mechanisms for sharing practice need to improve, and professional organisations including royal colleges must be doing that. Equal focus should be on removing the barriers to spreading the changes, and ensuring they are contextualised to local systems.

\section{Continuing to explore and develop}

The Q community is a network of 3,000 people who are active in health and care improvement across the UK, they all see the need to improve improvement. In the recent $\mathrm{Q}$ exchange funded programmes there was particular emphasis on working more effectively with patients, behavioural aspects of change, creating more effective networks and integrating methods from 
other disciplines. There are similar networks of innovators and researchers all aiming to improve the delivery of healthcare. Connecting these different tribes who have the same aims, and sometimes similar methods, will improve improvement through learning, understanding and enabling spread.

The Royal College of Physicians' Quality Improvement programme aims to be at the forefront of improving improvement by sharing good practice, supporting and learning with teams delivering improvement and working with others to develop better and more rigorous methods. Publication of new practice and good practice that can be spread, and learning from doing improvement even when it hasn't been successful will be enabled through Future Healthcare Journal.

Dr John Dean Clinical director of quality improvement and patient safety, Royal College of Physicians

\section{References}

1 Dixon-Woods M, Martin GP. Does quality improvement improve quality? FHJ 2016;3:191-4.
2 Dixon-Woods M. Harveian Oration 2018: Improving quality and safety in healthcare. Clin Med 2019;19:47-56.

3 Dixon-Woods M. How to improve healthcare improvement - an essay by Mary Dixon-Woods. BMJ 2019;367:I5514.

4 The Health Foundation. Quality improvement made simple: What everyone should know about health care quality improvement. London: The Health Foundation; 2013.

5 Batalden PB, Davidoff F. What is 'quality improvement' and how can it transform healthcare? Qual Saf Health Care 2007;16:2-3.

6 Jun GT, Ward J, Morris Z, Clarkson J. Health care process modelling: which method when? Int ] Qual Health Care 2009;21:214-24.

7 Taylor MJ, McNicholas C, Nicolay C et al. Systematic review of the application of the plan-do-study-act method to improve quality in healthcare. BMJ Qual Saf 2014;23:290-8.

8 Ogrinc G, Davies L, Goodman D et al. SQUIRE 2.0 (Standards for QUality Improvement Reporting Excellence): revised publication guidelines from a detailed consensus process. BMJ Qual Saf 2016;25:986-92.

9 Care Quality Commission. Quality improvement in hospital trusts: Sharing learning from trusts on a journey of QI. Newcastle: CQC; 2018.

\section{Supervising quality improvement projects} A guide for supervisors

\begin{abstract}
This online resource has been developed by the Royal College of Physicians (RCP) to help clinicians from any specialty to identify learning opportunities relating to quality improvement, to support and guide their trainees throughout their quality improvement projects.
\end{abstract}

For more information, please email: elearning@rcplondon.ac.uk

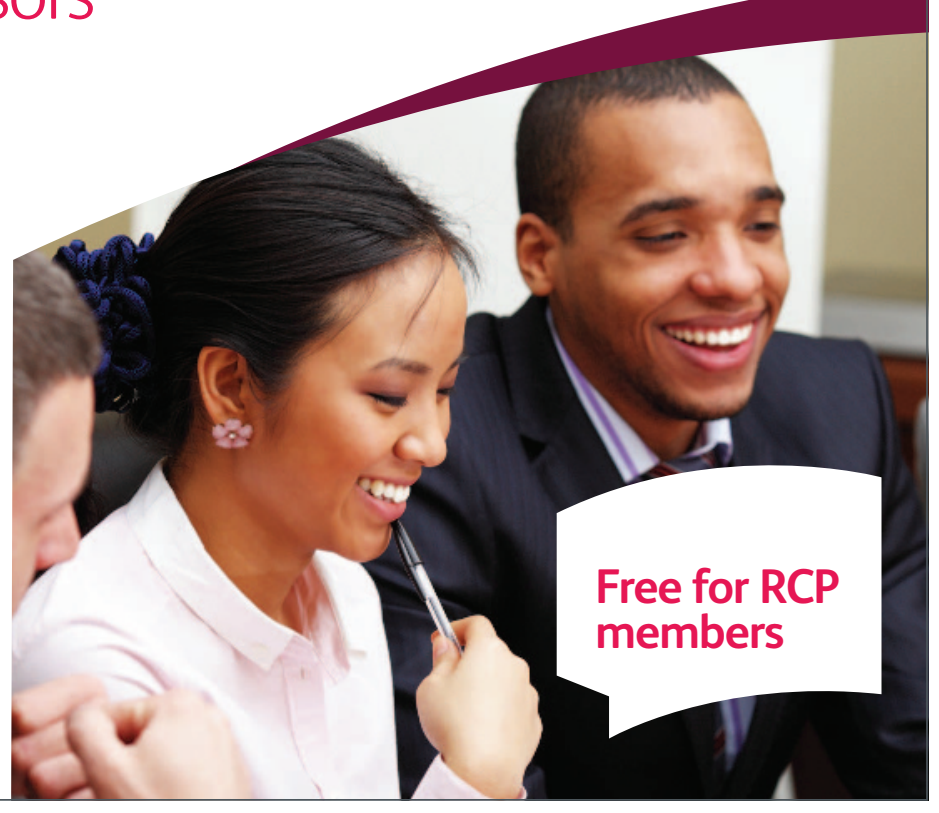

